\title{
THE END OF THE DARK AGES: PROBING THE REIONIZATION OF THE UNIVERSE WITH HST AND JWST
}

\author{
N. Panagia [ESA/STScI], M. Stiavelli \& S.M. Fall [STScI] \\ panagia@stsci.edu; mstiavel@stsci.edu; fall@stsci.edu
}

\begin{abstract}
Limiting the number of model-dependent assumptions to a minimum, we discuss the detectability of the sources responsible for reionization with existing and planned telescopes. We conclude that if reionization sources are UV-efficient, minimum luminosity sources, then it may be difficult to detect them before the advent of the James Webb Space Telescope (JWST). The best approach before the launch of JWST may be either to exploit gravitational lensing by clusters of galaxies, or to search for strong Ly- $\alpha$ sources by means of narrow-band excess techniques or slitless grism spectroscopy.
\end{abstract}

Keywords: cosmology: reionization; high- $z$ galaxies

\section{Introduction}

Motivated by recent evidence that the epoch of reionization of hydrogen may have ended at a redshift as low as $z \approx 6$ (e.g., Becker et al. (2001); Fan et al. (2002)), we have considered the detectability of the sources responsible for this reionization. The main idea is that reionization places limits on the mean surface brightness of the Population of reionization sources. We have defined a family of models characterized by two parameters: the Lyman continuum escape fraction $f_{c}$ from the sources, and the clumpiness parameter $C$ of the intergalactic medium. The minimum surface brightness model corresponds to a value of unity for both parameters. A maximum surface brightness is obtained by requiring that the reionization sources do not overproduce heavy elements. Our general approach is applicable to most types of reionization sources, but in specific numerical examples we focus on Population III stars, because they have very high effective temperatures and, therefore, are very effective producers of ionizing UV photons (e.g., Panagia et al. (2003)). Our mean surface brightness estimates are compared to the parameter space that can be probed by existing and future telescopes, in order to help planning the 


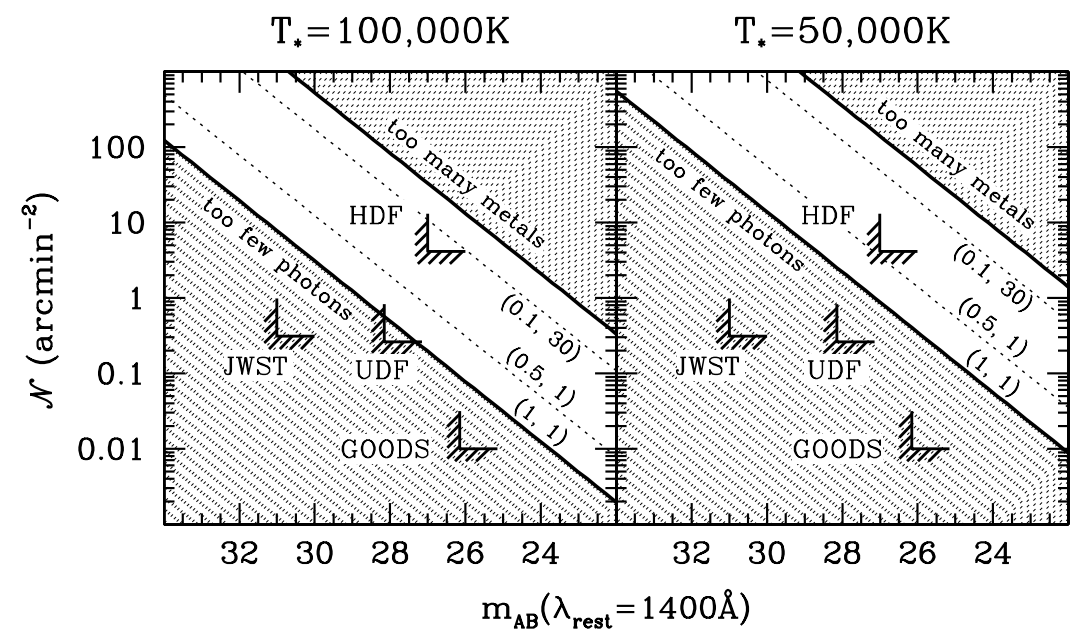

Figure 1. The loci of surface density vs apparent $\mathrm{AB}$ magnitude for identical reionization sources that are either Population III (left hand panel) or Population II stars (right hand panel).

most effective surveys. A full account of our work can be found in Stiavelli, Fall \& Panagia (2003).

\section{Results and Discussion}

In Figure 1 we show the loci of the mean surface brightness of identical reionization sources as a function of their observable $A B$ magnitude. The left panel refers to Population III sources with effective temperature of $10^{5}$ $\mathrm{K}$, the right panel to Population II reionization sources with effective temperature $5 \times 10^{4} \mathrm{~K}$. In both panels, the lower solid line represents the minimum surface brightness model, $(1,1)$, while the upper solid line represents the global metallicity constraint $Z \leq 0.01 Z_{\odot}$ at $z=6$. The thin dotted lines represent the $(0.5,1)$ and $(0.1,30)$ models. The non-shaded area is the only one accessible to reionization sources that do not overproduce metals. The Lshaped markers delimit the quadrants (i.e., the areas above and to the right of the markers) probed by the GOODS/ACS survey (Dickinson and Giavalisco (2003)), the HDF/HDFS NICMOS fields (Thompson et al. (1999); Williams et al. (2000)), the Ultra Deep Field (UDF) and by a hypothetical ultra-deep survey with JWST.

In Figure 2 we show the expected cumulative surface density distributions for reionization sources with a variety of luminosity functions. In each panel, the upper solid line represents the global metallicity constraint. The lower solid line represents the minimum surface brightness model, $(1,1)$. The thin dotted 


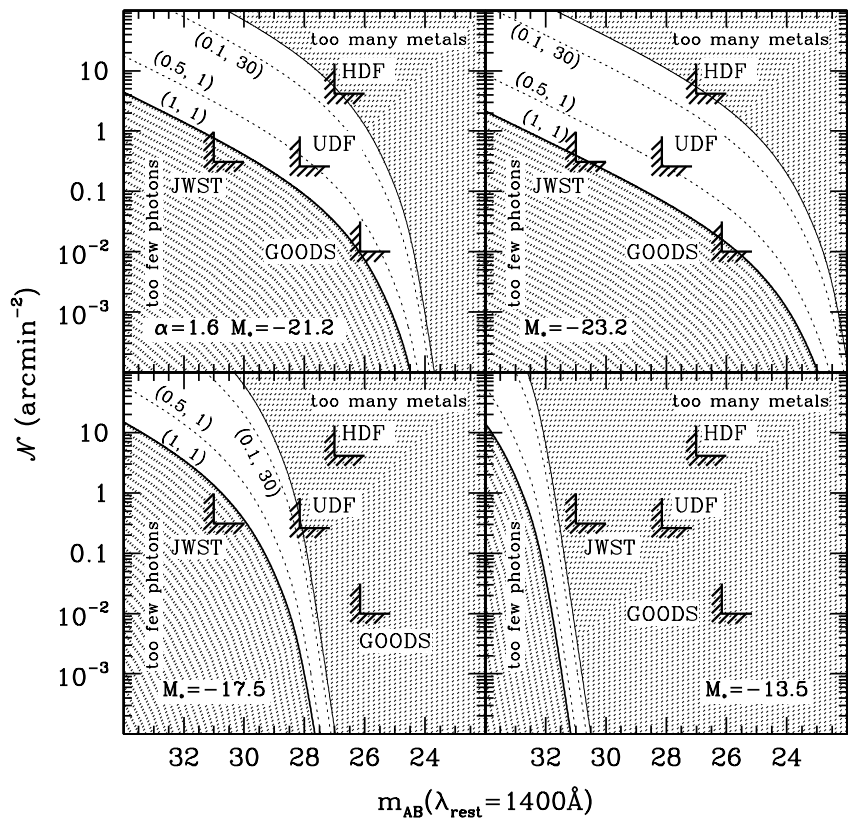

Figure 2. The cumulative distribution of the surface density vs apparent $\mathrm{AB}$ magnitude of reionization sources with luminosity functions with different knees.

lines give the luminosity function for the $(0.5,1)$ and the $(0.1,30)$ models. The L-shaped markers delimit the quadrants probed by the GOODS/ACS survey, the HDF and HDFS NICMOS fields, the UDF, and an ultra-deep survey with JWST, respectively.

From these results it appears that if reionization is caused by UV-efficient, minimum surface brightness sources, the non-ionizing continuum emission from reionization sources will be difficult to detect before the advent of JWST. On the other hand, if the sources of reionization were not extremely hot Population III stars but cooler Population II stars or AGNs, they would be brighter by 1-2 magnitudes and thus they would be easier to detect.

Finally, Figure 3 presents the required surface density as a function of the Ly- $\alpha$ line flux of reionization sources. The left hand panel shows the loci of identical sources for two different $\left(f_{c}, C\right)$ models (thin dotted lines). The top solid line identifies the global metallicity constraint. The right hand panel illustrates two different luminosity functions. The solid line refer to a luminosity function identical to that of $z=3$ Lyman break galaxies, (i.e., $M_{*, 1400}=$ -21.2 and $\alpha=1.6$, while the dashed line refers to a luminosity function with $M_{*, 1400}=-17.5$ and a slope identical to the local Universe slope, $\alpha=1.1$. In both panels, the L-shaped marker identifies the quadrant probed by a narrow- 


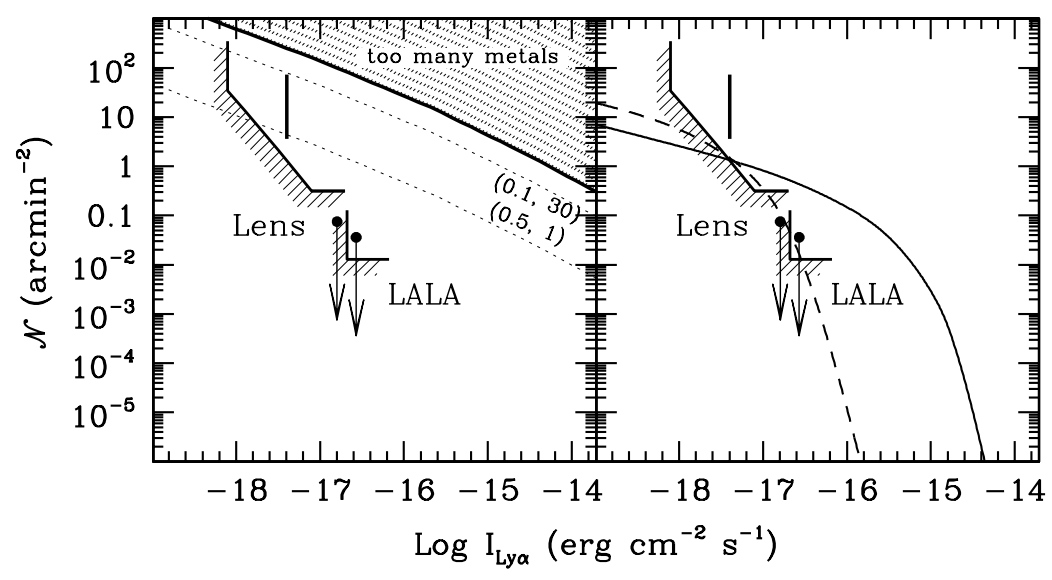

Figure 3. Surface density vs Ly- $\alpha$ line flux of reionization sources.

band excess survey at $z \geq 6$ (LALA survey, Rhoads and Malhotra (2001)). The oblique marker labeled Lens represents a hypothetical 100-orbit survey with the ACS grism on a cluster of galaxies to exploit gravitational amplification. The solid bar represents the density estimated from the detection at $z=6.56$ by $\mathrm{Hu}$ et al. (2002) while the two points with down-pointing arrows represent their upper limits.

It appears that searches based on narrow-band excess techniques or slitless grisms would be promising and might lead to the detection of the reionization sources within this decade.

\section{References}

Becker, R.H., et al. 2001, AJ, 122, 2850

Fan, X., et al. 2002, AJ, 123, 1247

Dickinson, M., \& Giavalisco, M. 2003, in the proceedings of the ESO/USM Workshop "The Mass of Galaxies at Low and High Redshift", eds. R. Bender \& A. Renzini, p. 324

Hu, E.M., et al., 2002, ApJ, 568, L75

Panagia, N., Stiavelli, M., Ferguson, H. \& Stockman, H.S., 2003, in preparation [see also astro$\mathrm{ph} / 0209278$ and /0209346]

Rhoads, J.E. \& Malhotra, S., 2001, ApJ, 563, L5

Stiavelli, M., Fall, S.M., \& Panagia, N., 2003, ApJ, in press

Thompson, R., Storrie-Lombardi, L., Weymann, R.J., Rieke, M.J., Schneider, G., Stobie, E., \& Lytle, D., 1999, AJ, 117, 17

Williams, R.E., et al., 2000, AJ, 120, 2735 